\title{
Diffuse peritonitis and chronic ascites due to infection with Chlamydia trachomatis in patients without liver disease: new presentation of the Fitz-Hugh-Curtis syndrome
}

\author{
U A MARBET, G A STALDER, J VÖGTLIN, J LOOSLI， A FREI， B ALTHAUS， K GYR
}

\begin{abstract}
Two women were admitted for increasing abdominal pain, vaginal discharge, and severe or moderate chronic ascites. Diffuse peritonitis without evidence of liver disease was found in both cases, and in one the ascites and vaginal discharge contained Chlamydia trachomatis. Both patients responded to doxycycline, and this and the laboratory findings pointed strongly to $\mathrm{C}$ trachomatis as the aetiological agent.

C trachomatis may cause severe peritoneal infections with chronic ascites formation in the absence of liver disease in women with the Fitz-Hugh-Curtis syndrome. Prompt diagnosis and antibiotics lead to rapid cure.
\end{abstract}

\section{Introduction}

Chronic ascites in patients without concomitant liver disease has apparently not been reported in the Fitz-Hugh-Curtis syndrome. ${ }^{12}$ We therefore describe two patients with the syndrome who presented with diffuse peritonitis and ascites due to infection with Chlamydia trachomatis.

\section{Case 1}

A 28 year old woman was admitted with a history of increasing abdominal pain and ascites for five months after noticing a whitish vaginal discharge associated with using an intrauterine device.

\footnotetext{
Division of Gastroenterology, Department of Internal Medicine, University Hospital, CH-4031 Basle, Switzerland

U A MARBET, MD, staff physician

G A STALDER, MD, staff physician

J VÖGTLIN, MD, staff physician

J LOOSLI, MD, staff physician

A FREI, MD, staff physician

B ALTHAUS, MD, staff physician

K GYR, MD, MPH\&TM, staff physician

Correspondence to: Dr Marbet.
}

Examination showed the patient to be in poor general condition with severe ascites. There were no gynaecological abnormalities and liver enzyme activities and prothrombin time were normal. The table gives the laboratory findings. Tuberculosis was excluded. Ascitic fluid contained $76 \mathrm{~g}$ protein per litre, few leucocytes, and no bacteria on culture. Laparoscopy showed multiple fibrous adhesions of the whole peritoneal cavity including the liver surface with fibrous plaques on both adnexa and large ascites. Biopsy of the peritoneum yielded fibrous tissue without inflammation. Biopsy of the liver showed non-specific reactive hepatitis. The skin was anergic to purified protein derivative of tuberculin and to seven common intracutaneous antigens (Multitest, Mérieux). Nothing abnormal was found on radiography and biopsy of the small intestine, computed tomography of the abdomen, smear and biopsy of the bone marrow, or bacterial and parasitological studies of the stool. Cultures of cervical and ascitic fluid grew no pathogens. Cultures for $C$ trachomatis were taken only after starting treatment and also gave negative results.

Case 1. Chronological sequence of antibody titres against trachomatis and pathological laboratory data

\begin{tabular}{|c|c|c|c|c|}
\hline & \multicolumn{2}{|c|}{1984} & \multicolumn{2}{|c|}{1985} \\
\hline & 16 March & 15 June & 1 April & 20 November \\
\hline Complement fixation test & 1240 & 1120 & 1120 & 160 \\
\hline Immunofluorescent IgG & 12560 & 12560 & 12560 & 12560 \\
\hline Immunofluorescent IgM & 1320 & 180 & 180 & 180 \\
\hline Immunofluorescent IgA & 11280 & 11280 & 1640 & 1640 \\
\hline Erythrocyte sedimentation & & & & \\
\hline rate ( $\mathrm{mm}$ in first hour) & 108 & 15 & 13 & 4 \\
\hline Platelet count $\left(\times 10^{9} 1\right)$ & 927 & 478 & 377 & 417 \\
\hline Serum albumin $(\mathrm{g} / \mathrm{l})$ & 26 & 42 & 44 & 43 \\
\hline
\end{tabular}

The intrauterine device was removed and the patient treated with doxycycline $200 \mathrm{mg}$ daily for six weeks. She recovered rapidly, the pain and ascites disappearing completely. Four months later, two weeks after insertion of a new intrauterine device, she had a recurrence of abdominal pain with formation of ascites. Results of bacterial investigations were again negative. She responded well to a second course of doxycycline $200 \mathrm{mg}$ daily for four weeks, followed by $100 \mathrm{mg}$ daily for five months, and more than one year later remained symptom free. Helper/suppressor $\mathrm{T}$ cell ratio and lymphocyte responses to different plant mitogens were normal. No antibodies to human $\mathrm{T}$ cell lymphotropic virus type III were detected at follow up but her skin reactivity remained anergic. 
Case 2

A 26 year old waitress with chronic alcohol abuse was admitted because of increasing, predominantly left sided abdominal pain for three weeks, formation of ascites, and yellow vaginal discharge. Laboratory findings were normal except for a sedimentation rate of $44 \mathrm{~mm}$ in the first hour and platele count of $511 \times 10^{9} / 1$. Laparoscopy showed severe diffuse fibrinous peritonitis, most pronounced in the right upper quadrant, and a moderate amount of ascites. $C$ trachomatis was cultured from the ascites and vaginal discharge. The patient was treated successfully with doxycycline $200 \mathrm{mg}$ daily for 10 days.

\section{Comment}

Shabot and colleagues found $C$ trachomatis in ascitic fluid of patients with chronic liver disease but were unable to say whether the organism was pathogenic in those cases. ${ }^{3}$ Diffuse peritonitis and ascites formation in our patients without chronic liver disease were definitely due to an infectious agent, as shown by the rapid response to antibiotic treatment. Preceding genital infection and manipulation of the uterus are classic features of the Fitz-Hugh-Curtis syndrome. $C$ trachomatis was the most likely aetiological agent in both patients.
In case 2 the organism was cultured from the cervical secretion and ascitic fluid and in case 1 the patient showed a fourfold decline of immunofluorescent IgM antibodies after treatment. ${ }^{5}$

These two patients show that $C$ trachomatis may cause severe chronic peritoneal infections with chronic ascites formation. Timel diagnosis and correct treatment lead to rapid cure.

We thank Dr L Matter, of the Institute of Microbiology, St Gallen, for the serological work up, Dr M Zimmerli for advice, and Mrs C Frei for thed manuscript.

\section{References} 1 Oriel JD. Epidemiology of genital chlamydia infections. Infection 1982;10(suppl):532-9. Chlamydia trachomatis as possible cause of peritonitis and perihepatitis in young women Br Med F 1978;i:1022-4.

3 Shabot JM, Roark GD, Truant AL. Chlamydia trachomatis in the ascitic fluid of patients wite chronic liver disease. Am f Gastroenterol 1983;78:291-4.

4 Onsrud $M$. Perihepatitis in pelvic inflammatory disease-association with intrauterine contracepen tion. Acta Obstet Gynecol Scand 1980;59:69-71.

5 Treharne JD. Chlamydia trachomatis: serological diagnosis. Infection 1982;10(suppl 1):525-31. $\overrightarrow{0}$ (Accepted 30 April 1986)

\title{
Cigarette smoking and risk of premature stroke in men and women
}

\author{
RUTH BONITA, ROBERT SCRAGG, ALISTAIR STEWART, RODNEY JACKSON, \\ ROBERT BEAGLEHOLE
}

\begin{abstract}
A case-control study was carried out of the relation between cigarette smoking and hypertension and stroke. A total of 132 cases of stroke ( 79 in men, 53 in women) identified as a part of a population based register were compared with 1586 controls (1017 men, 569 women) from a survey of cardiovascular risk factors conducted in the same population. Cigarette smokers had a threefold increase in the risk of stroke compared with current non-smokers. This association remained significant after adjusting for hypertension. Those who both smoked and had hypertension had an increased risk of stroke of almost 20 -fold compared with those who neither smoked nor had hypertension.

Overall, in this population roughly $37 \%$ of stroke events may be attributed to cigarette smoking and $36 \%$ to hypertension.
\end{abstract}

\section{Introduction}

High blood pressure is consistently reported as the most important known risk factor for stroke. ${ }^{1-4}$ Other risk factors such as cigarette smoking or serum lipid, blood glucose, and plasma fibrinogen concentrations have shown varying associations. ${ }^{34}$ In particular,

Department of Medicine, School of Medicine, University of Auckland, Auckland, New Zealand

RUTH BONITA, MPH, PHD, research officer

Department of Community Health and General Practice, School of Medicine,

University of Auckland, Auckland, New Zealand

ROBERT SCRAGG, MB, PHD, senior lecturer in epidemiology

ALISTAIR STEWART, BSC, DIPSC, biostatistician

RODNEY JACKSON, мB, DCOMH, National Heart Foundation of New

Zealand training fellow in epidemiology

ROBERT BEAGLEHOLE, MD, FRACP, associate professor of epidemiology

Correspondence to: Dr Bonita. many studies have failed to identify cigarette smoking as significant risk factor for stroke. When a positive association has been found this has usually been confined to young men. ${ }^{56}$

We present a population based case-control analysis of the association of cigarette smoking and hypertension with strok in both men and women. The proportions of stroke events thaf could be attributed to smoking and hypertension are also estimate $\bar{\Phi}$ as this does not appear to have been done before. In addition, the study investigates the possible synergism between smoking an hypertension.

\section{Subjects and methods}

Cases of stroke were identified from a population based cardiovascula disease register, in which a 50\% random sample of all new episodes of strokeె in Auckland was studied in the year ending 1 March 1982. The study ared (population 829545) encompassed one quarter of the total population of New Zealand. Full details of the case finding methods have been published.o The diagnostic criteria and methods were based on the World Healtb Organisation guidelines. ${ }^{8}$ Stroke was defined as the rapid onset of focat. neurological deficit, presumably of vascular origin, lasting 24 hours oN longer or leading to death. Patients with subarachnoid haemorrhage wer: excluded.

Information was obtained from patients using a standard questionnaire a? soon after the event as possible. If the patient had died the samo questionnaire was administered to a close relative after six weeks. Detailक्ष about treatment for high blood pressure and cigarette smoking werẹ collected. Current pharmacological treatment for high blood pressure waso used as a surrogate measure of hypertension. A current smoker was define $\Phi$ as a person smoking at least one cigarette a day.

Controls for the analysis came from a population based survey if Auckland, the sampling methods and results of which have been reported. $\cong$ Briefly, 1017 men and 569 women aged 35-64 were interviewed betweer January and July 1982 after being randomly selected from the $198 \mathrm{~b}$ Auckland general electoral rolls (response rate $82 \%$ ). The questionnairo included the same questions about blood pressure history and cigarette smoking as were asked of the patients with stroke.

Analyses were restricted to events that occurred in men and women aged 